\title{
Material-integrated composite humidity sensors for condition monitoring of fiber-reinforced plastics
}

Jörg Martin ${ }^{1)}$, Kuldeep Shetty ${ }^{2)}$, Nadine Reimann ${ }^{3)}$, Stephan Neukirchner ${ }^{4)}$, Uta Fügmann'2), Heike Illing-Günther ${ }^{4}$, Daisy Nestler ${ }^{4) 5}$, Arved C. Hübler ${ }^{2)}$, Klaus Nendel ${ }^{3)}$, Lothar Kroll(5), Thomas Otto ${ }^{1 / 6)}$

1) Department Multi Device Integration, info@enas.fraunhofer.de, Fraunhofer Institute for Electronic Nano Systems ENAS, Technologie-Campus 3, 09126 Chemnitz, Germany

2) Institute for Print- und Media Technology, pminfo@mb.tu-chemnitz.de, Technische Universität Chemnitz, Reichenhainer Straße 70, 09126 Chemnitz, Germany

3) Professorship of Materials Handling and Conveying Engineering, klaus.nendel@mb.tuchemnitz.de,Technische Universität Chemnitz, Reichenhainer Street 70, 09126 Chemnitz, Germany

4) Saxon Textile Research Institute e. V., stfi@stfi.de, An-Institute Technische Universität Chemnitz, Annaberger Straße 240, 09125 Chemnitz, Germany

5) Department of Lightweight Structures and Polymer Technology, slk@mb.tu-chemnitz.de, Technische Universität Chemnitz, Reichenhainer Straße 31/33, 09126 Chemnitz, Germany

6) Center for Microtechnologies, info@zfm.tu-chemnitz.de, Technische Universität Chemnitz, Reichenhainer Straße 70, 09126 Chemnitz, Germany

\section{Keywords}

Humidity Sensor, Nanocomposite, Flexographic Printing, Lightweight Structures, Soutache Embroidery.

\begin{abstract}
Penetrating water in fiber-reinforced plastics can alter the mechanical properties considerably. To avoid potential resulting failure of the component, we propose continuous monitoring of the humidity inside the material by highly-sensitive humidity sensors based on nano- or microcomposites. Here we report on the inline-capable fabrication and integration of humidity sensors in glass fiber-reinforced polyamide (GF-PA6). Mean water concentrations of less than $0.5 \mathrm{wt}$. \% have been clearly determined inside the laminate.
\end{abstract}

\section{Introduction}

Fiber-reinforced plastics (FRP) offer excellent mechanical properties at low weight. Hence, such materials are ideally suited to reduce energy consumption and $\mathrm{CO}_{2}$ emission, especially in the field of mobility. However, mechanical properties of FRPs can be altered drastically if water is absorbed by the material. For instance, a reduction in flexural strength and in shear fracture toughness has been detected for glass and carbon fiber-reinforced polymer composites after immersion in deionized and seawater [1, 2]. Here only the natural water uptake by polymers was investigated. Water, which may penetrate into the structure directly, e.g. via micro cracks because of a shallow damage, will intensify the degradation. Delamination, loss of stiffness and finally complete failure of the structure might be the consequences.

To avoid unpredictable failure by water absorption, we propose to monitor the water content of fiberreinforced materials continuously via incorporated thin-film humidity sensors. However, there are several demands on the sensor configuration. These include a high sensitivity of the sensors as well as easy scalability to allow for monitoring of larger structures and the detection of humidity distributions. In addition, the capability of in-line fabrication and integration should be given, including the electrical connection of the sensors in the laminate. Finally, the sensors must not change the mechanical properties of the structural element.

Digital Object Identifier: http://dx.doi.org/10.21935/tls.v1i2.88 www.lightweight-structures.de 
For the determination of humidity, many different measurement techniques have been developed, whereby the resistive and capacitive method is predominantly used for electronic readout and further data processing [3]. Modern thin-film sensors usually use polymers, porous ceramics or even paper as sensitive dielectrics [4,5]. Pure ceramics imply several advantages, e.g. thermal and chemical resistance, but they cannot be integrated in laminates in a reasonable and economic way because of their non-bendable and brittle characteristics.

In contrast, polymeric nano- and microcomposites offer the opportunity to combine the advantages of ceramics and polymers and minimize the disadvantages. Innovative humidity sensors by means of nanocomposites can be prepared with a large scale on rigid, bendable or even curved surfaces employing a variety of electrode structures [6-8].

Hereafter, we present results according to the preparation, characterization and integration of micro-/nanocomposite humidity sensors into FRPs. The layout and preparation technology of the sensors by means of flexographic printing is described in detail, as well as strategies for electrical connection via embroidery technology and integration in glass fiber-reinforced polyamide composites (GF-PA6). Finally, characteristics of bare and integrated sensors are discussed with respect to sensitivity and in the context of diffusion and water absorption by the laminate.

\section{Materials and methods}

\subsection{Layout of composite humidity sensors}

The manufactured nano-/microcomposite humidity sensors work on the capacitive readout principle based on a hygroscopic dielectric layer. This dielectric consists of nanoporous silica particles of a size range of 2-25 $\mu \mathrm{m}$ (Sigma Aldrich), which are embedded in a polyvinyl butyral (PVB, Mowital B $16 \mathrm{H}$, kuraray) matrix. Since the dielectric permittivity of water is much larger than that of the polymer or porous silica ( $\varepsilon_{\text {water }}=79.1$ compared to $\varepsilon$ silica $~ 1.2-3.0$, depending on porosity [9], and $\varepsilon$ PVB $\sim 4.5$ [10]), adsorbed water changes the dielectric permittivity drastically, and hence the capacitance of the setup.

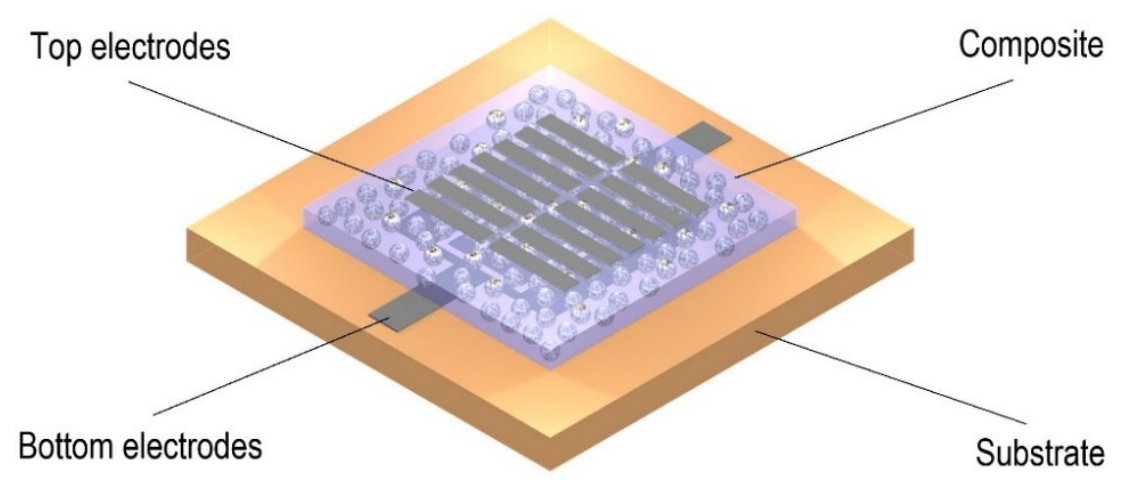

Figure 1: Illustration of capacitive composite humidity sensors

In Figure 1, the sensor layout is depicted. The moisture-sensitive dielectric is sandwiched between bottom and intermittent top electrodes. In fact, the layout contains two groups of seven parallel capacitances, which are connected in series. The electrodes and the dielectric layer were applied on a (flexible) polyimide film (Kapton® CR, DuPont, thickness: $50 \mu \mathrm{m}$ ) via flexographic printing. Kapton® foil was chosen as substrate because of its high temperature stability and low expansion, which are prerequisites for handling of the thermal and mechanical loads during the lamination process. It is worth to note, that only the (separated) ground electrodes are contacted to the measuring voltage, leaving the top electrodes at floating potential. From electronics point of view, one should avoid floating electrodes because of easy coupling to interfering signals. But with careful analysis of the sensor signal, this configuration can enhance the detectability of absorbed water. Because changes of permittivity in the surrounding of the sensor influence the floating electrode potential, and thus the sensor capacitance, the sensitivity of the sensor can be further enhanced. So it is possible to detect water already outside the sensor structure, as will be discussed in chapter 3. 


\subsection{Sensor fabrication, electrical contacting and integration in laminates}

Humidity sensors were printed on flexographric printing process as shown in Figure 2. Anilox roller screened with cells pick the ink and transfers it to the raised areas of the flexible plate ("Imaging areas") with the pattern to be printed. The areas on the plate which are lower than the raised are the nonimaging areas of the plate. The pattern is printed on the substrate on contact with the raised areas of the plate.

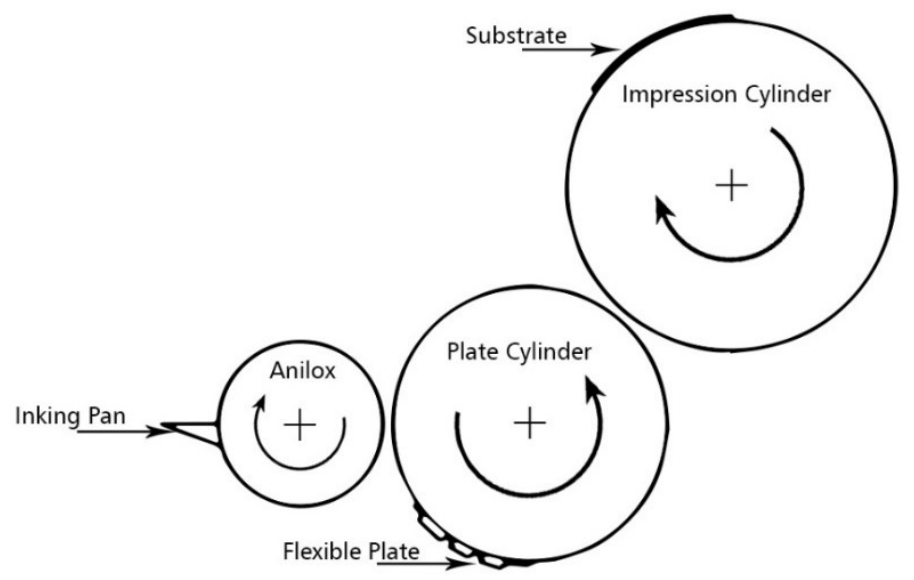

Figure 2: Flexograhic printing process

For the bottom electrode, a silver nano ink Jet-001T by Kunshan Hisense Electronics was used for its high conductivity and low surface peaks to avoid contact between top and bottom electrode through the dielectric. Printed bottom electrode pattern was dried in a hot air oven at $130{ }^{\circ} \mathrm{C}$ for 5 mins. Multiple layers of dielectric ink were printed 'dry on dry' consisting of $10 \mathrm{wt}$. \% solid (6 wt. \% polyvinyl butyral + $4 \mathrm{wt}$. \% silica) in 1-butanol to achieve no contact between the top and bottom electrodes with minimal thickness of dielectric layer. Each dielectric layer was dried in the hot air oven at $120^{\circ} \mathrm{C}$ for 5 mins before printing the next layer. The top electrodes were printed of poly(3,4-ethylenedioxythiophene) polystyrene sulfonate (PEDOT:PSS) Clevios Sv3 ink or silver-based ink DuPont 5028 with 15 vol. \% of butyl glycol (2 layers). PEDOT:PSS was dried in the hot air oven at $120^{\circ} \mathrm{C}$ for 5 mins, whereas each layer of DuPont 5028 at $130{ }^{\circ} \mathrm{C}$ for 5 mins. 7 layers of dielectric ink were printed for sensors with top electrode of PEDOT:PSS, whereas sensors with DuPont 5028 had 10 layers of dielectric ink. All the layers were printed on lab scale flexographic printing machine Flexiproof 100 with the settings mentioned in the below Table 1.

Table 1: Print settings on Flexiproof printing machine for various functional layers

\begin{tabular}{cccccc}
\hline Layer & $\begin{array}{c}\text { Anilox } \\
\text { enaravina } \\
\text { (Lines/cm) }\end{array}$ & $\begin{array}{c}\text { Anilox volume } \\
\left(\mathbf{c m}^{3} / \mathbf{m}^{2}\right)\end{array}$ & $\begin{array}{c}\text { Anilox } \\
\text { Pressure } \\
\text { (a. u. })\end{array}$ & $\begin{array}{c}\text { Impression } \\
\text { Pressure } \\
\text { (a. u. })\end{array}$ & $\begin{array}{c}\text { Speed } \\
\text { (m/min) }\end{array}$ \\
\hline $\begin{array}{c}\text { Bottom } \\
\text { Electrode }\end{array}$ & 55 & 24.99 & 14 & 80 & 30 \\
$\begin{array}{c}\text { Dielectric } \\
\text { Layer }\end{array}$ & 30 & 46.7 & 14 & 80 & 60 \\
$\begin{array}{c}\text { Top Electrode: } \\
\text { PEDOT:PSS }\end{array}$ & 30 & 46.7 & 14 & 70 & 30 \\
$\begin{array}{c}\text { Top Electrode: } \\
\text { DuPont 5028 }\end{array}$ & 55 & 24.99 & 14 & 70 & 30 \\
\hline
\end{tabular}


Contacting of the sensors was carried out by embroidery technology. Since embroidery technology does not change the characteristics of the flexible printed sensors, this technology is best suited for in-line sensor integration. The embroidery head enables a flexible laying and fixation of suitable wires onto the GF-PA6 tape. With its three thread systems, it simultaneously guarantees the fixing and the electrical contacting of the sensor. The machine allows to place the wire for the contacting in two-dimensional level of the carrier material with very high precision.

The work was done using the stitching machine SGW 0100-1375 by ZSK Stickmaschinen $\mathrm{GmbH}$ (see Figure 3). It allows the in-line production by roll-to-roll. The automatic pneumatic clamping enables the continuous production of samples with multiple sensors with the following features:

- damage-free continuous processing by production from roll-to-roll,

- free choice (CAD) of the drive path with return to the starting point by degrees of freedom in $\mathrm{x}$, y direction,

- slight restriction by embroidery frame size and

- an enormous number of available stitching heads.
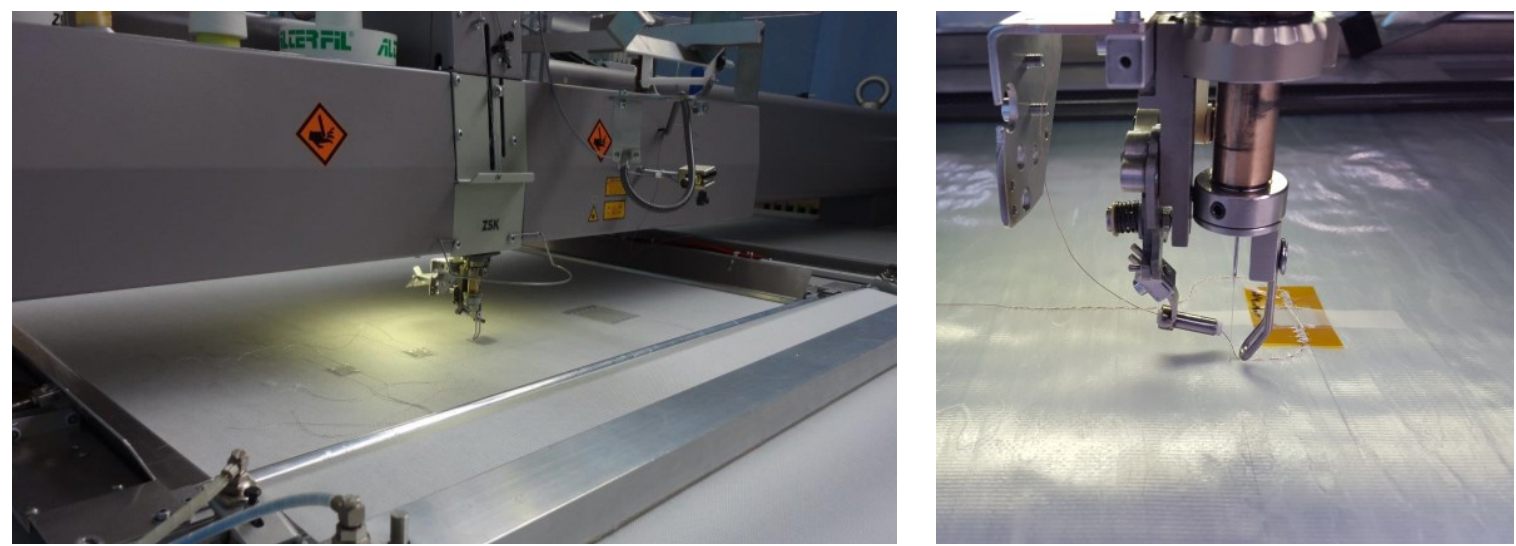

Figure 3: Stitching machine by ZSK Stickmaschinen $\mathrm{GmbH}$ with head for tailored placement

For checking the functionality, all sensors were tested before and after stitching. Further characterization took place after stitching and the process of consolidation in the climate chamber. To avoid the floating of surplus material of polyamide and the damage of the contact between wire and printed sensor during the pressing process, at some samples the copper wires close to the contact pads of the bottom electrodes are covered by conductive silver varnish and an additional layer of polyimide-tape (Figure 4).
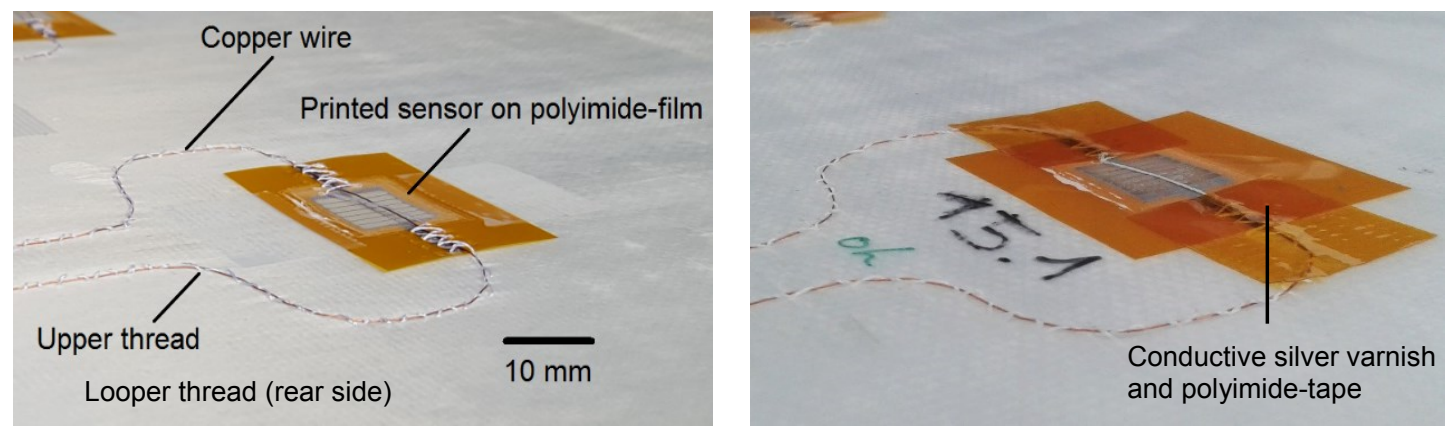

Figure 4: Composite humidity sensors on thermoplastic prepreg contacted via soutage embroidery. Images taken shortly after preparation, copper wires across the active sensor layers were separated later on.

Various samples of hybrid laminate were produced in a positive mold, each with a width and length of $170 \mathrm{~mm}$. Diverse layouts of layer structure were carried out. The consolidation process is an isobaric process with 26 bar in the first phase and continuous heat input of $285^{\circ} \mathrm{C}$. This high temperature was necessary to establish a very low viscosity of PA6. Subsequently, all the fibers were pre-consolidated within the PA-matrix. The temperature was maintained for a period of 2-3 $\mathrm{min}$ at a pressure of 36 bar. 
A pressure of 12 bar was maintained during the cooling process within 7 minutes at $60{ }^{\circ} \mathrm{C}$ and an additional waiting time until the sample could be demolded (see Figure 5). This guarantees the correct fiber-matrix adhesion and avoids delamination by residual stress.
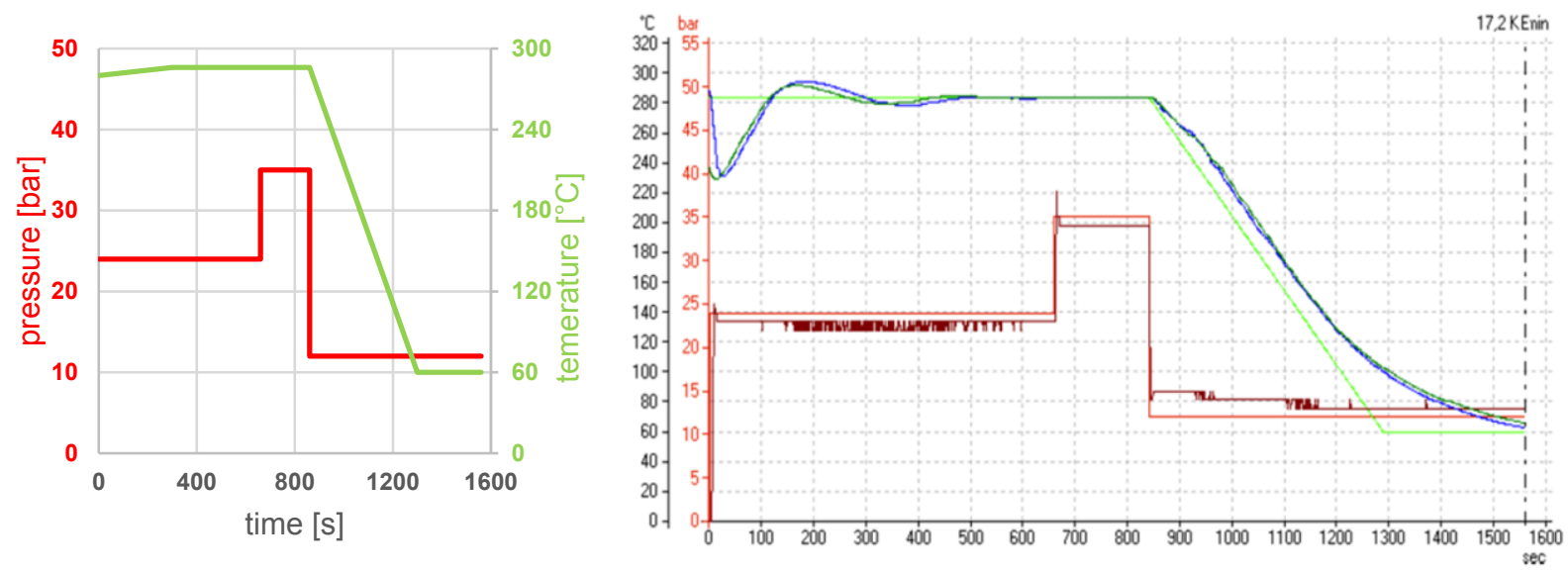

Figure 5: Projected course and testing protocol of a consolidation test

\subsection{Characterization}

Bare sensors have been characterized in a climatic chamber (KPK 200, Feutron). Humidity changing regimes were applied to the sensors and capacitance values were recorded with a LCR meter (HM 8118, Rohde \& Schwarz HAMEG). According to the defined measurement profile, the temperature was fixed at $50{ }^{\circ} \mathrm{C}$ and the relative humidity was adjusted between $10 \% \mathrm{r}$. h. and $90 \% \mathrm{r}$. h. in steps of $10 \%$. After each step of increasing or decreasing humidity, the value was held for at least 20 min, to allow the humidity to stabilize. After the measurement, capacitance humidity characteristics of the respective sensors have been extracted from the temporal records of humidity set point, actual humidity value and capacitance.

After consolidation of the thermoplastic laminate, samples with incorporated sensors of size $100 \mathrm{x}$ $70 \mathrm{~mm}^{2}$ were cut out (Figure 6, left). The copper wires were uncovered at the edge of the plates and contacted to copper pads via conducting epoxy glue. For measuring the sensor response on water uptake by the laminate, a continuous recording of capacitance and impedance has been carried out. Before the measurement, the samples have been dried in a vacuum drying chamber at $80{ }^{\circ} \mathrm{C}$ for 8 hours. After determining electrical reference values in air, the laminate was immersed into water at a temperature of $23{ }^{\circ} \mathrm{C}$ and capacitance/impedance were measured each 30 seconds for several hours with the LCR meter (Figure 6, right). Additionally, for investigation of water penetration, a piece of the same laminate without sensor was kept in water for more than 10 days and its weight was determined regularly by a lab balance (Voyager Pro, Ohaus).
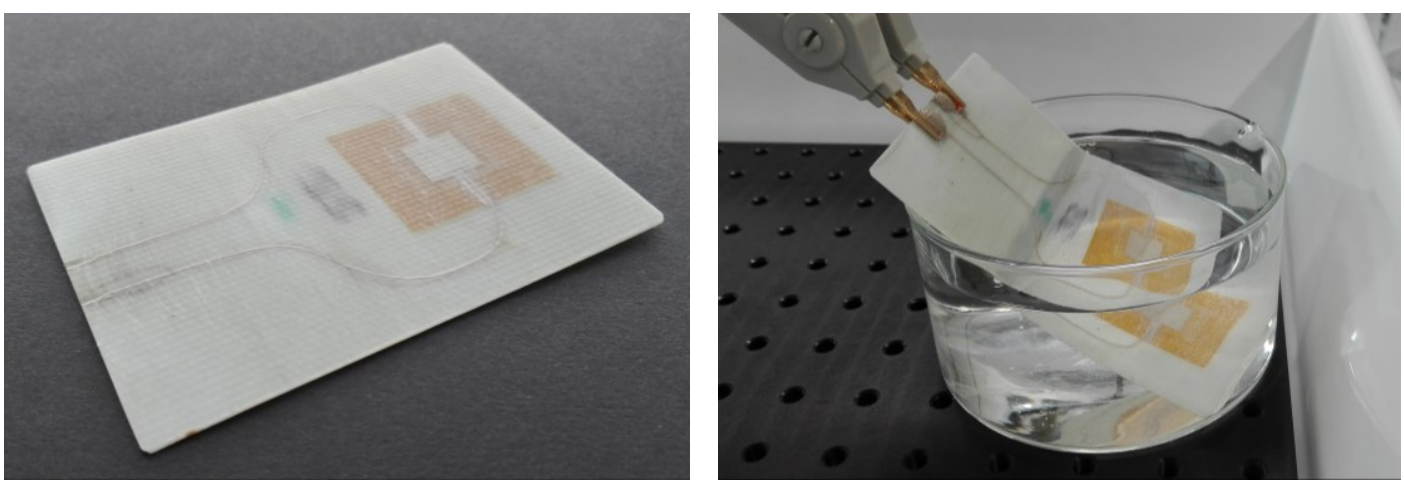

Figure 6: Thermoplastic laminate (GF-PA6) with incorporated composite humidity sensor (left) and measurement of sensor response on water uptake with sample immersed in water (right). 


\section{Results and discussion}

The sensitive elements in the sensor dielectric are nanoporous silica particles. Hence, one can expect that with increasing content of particles in the layer, the sensitivity of the sensors will increase too. Actually, a higher particle concentration leads to a higher number of particles at the surface interacting with the surrounding atmosphere and thus adsorbing more water. A higher sensitivity of samples with large volume concentrations of particles was demonstrated [7]. But on the other hand, too high a volume fraction turns the microcomposite into a fragile and crumply layer, not suitable for integration. It turned out that a volume concentration of $60 \%$ silica particles is the best compromise between sensitivity and mechanical stability of the layer.

In Figure 7, the sensor characteristics of printed composite sensors are shown. Sensor layout and thickness of dielectric layer are the same, the sensors differ only in the material of the top electrode, which is either silver or PEDOT:PSS. With more than an 8 fold increase of capacitance in a range of relative humidity between 10 and $90 \%$, the sensors exhibit a very high sensitivity. Moreover, both types display a similar behavior with a tending stronger non-linearity of the PEDOT:PSS sensor. We attribute this to the water-sensitivity of PEDOT:PSS itself, resulting in a reduced impedance of the top electrode with increasing relative humidity above $70 \%$ r. h. [11].

For the detection of water in a fiber-reinforced plastic material, the sensitivity of the sensor is the most important point, since already small amounts should be detected. The non-linearity is less critical and can be corrected with data processing. Consequently, both types of printed sensors are suited for integration.

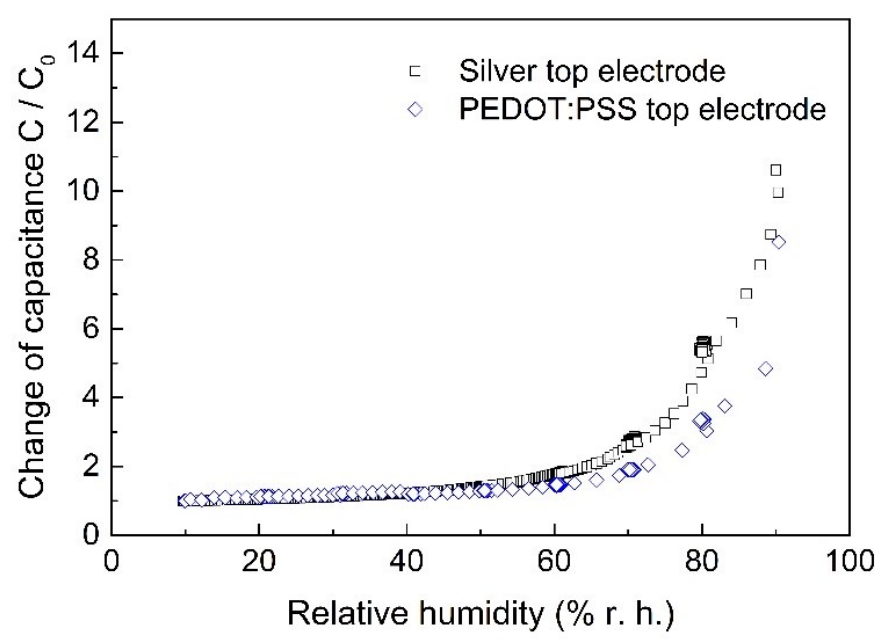

Figure 7: Change of capacitance as function of relative humidity for printed sensors with silver and PEDOT:PSS top electrodes

Several sensors of the series with silver top electrodes have been incorporated in GF-PA6 laminates to investigate the capability of water detection inside the material. For this, pieces of the laminate where immersed in water for several hours. A typical capacitance readout of an embedded composite sensor can be found in Figure 8 (left).

Instantaneously with water immersion, a step in capacitance from $90 \mathrm{pF}$ to nearly $105 \mathrm{pF}$ was measured ("Offset 1"), followed by a further slow increase. At an observation time of 30 hours, the sample was removed from the water, which resulted in a fast drop of capacitance values. After drying the outside of the sample, still an offset was present with respect to the initial capacitance value ("Offset 2"). Obviously, the second offset originates from water which is absorbed by the laminate. 

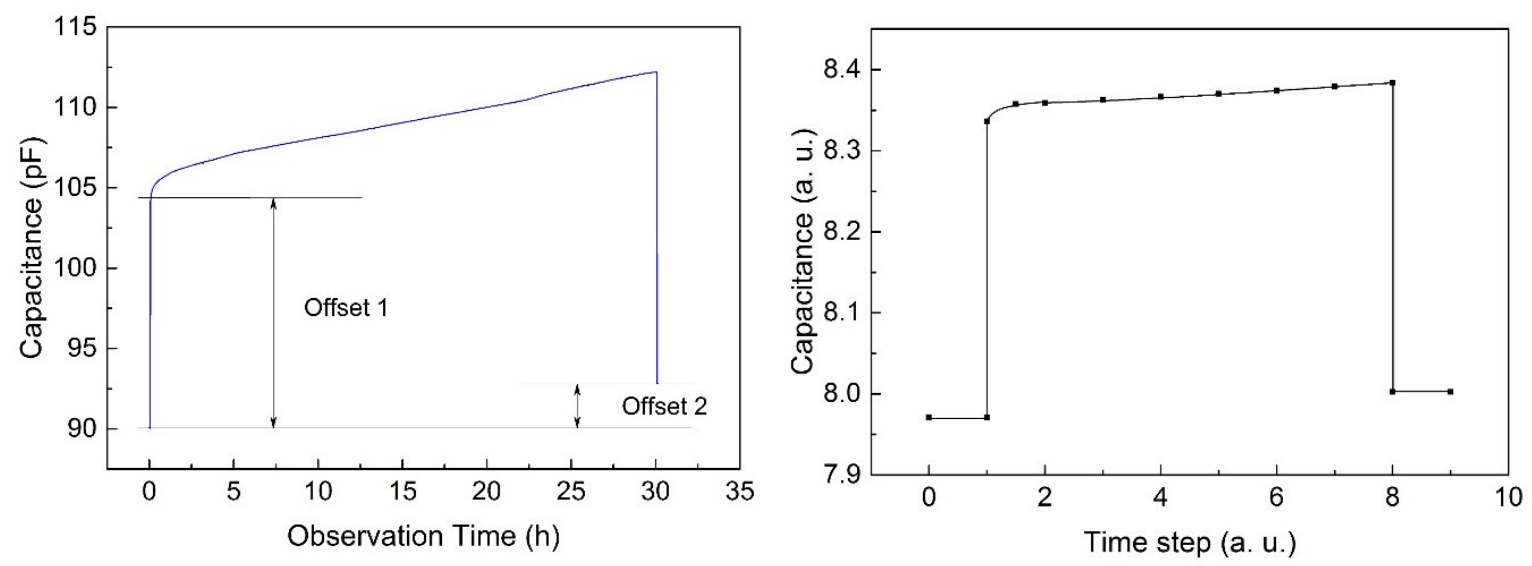

Figure 8: Capacitance of a GF-PA6 sample with integrated humidity sensor during water immersion (left), and FE-simulated capacitance-time-behavior of a corresponding generic sample (right)

To verify the recorded characteristics, a quasi-steady finite element (FE) simulation was carried out. Although a simplified water diffusion profile was used in the calculation, the measured curve could be reproduced quite well, as can be seen from the right graph in Figure 8. For instance, the capacitance steps at the beginning and the end were simulated by changing the permittivity of the surrounding from 1.0 to 79.1 (air to water) and vice versa. Further, the slow capacitance increase was replicated by a layer by layer change of the laminate permittivity from dry to wet condition (estimated values, dry: $\varepsilon=4.0$, wet: $\varepsilon=4.9$ ), starting at the surface of the laminate. Following this strategy, a further strong increase in capacitance was calculated when reaching the sensor dielectrics (not shown here). Hence, we can conclude that after an observation time of $30 \mathrm{~h}$ no significant water concentration is present at the position of the sensor.

Figure 9 shows the graph of an identical measurement with extended observation time. Again, steps in capacitance with respect to changes from air to water and back to air in the surrounding are clearly visible. Additionally, offset 2 is more pronounced than in Figure 8, suggesting a higher amount of absorbed water. Interestingly, even after 72 hours no saturation of the capacitance signal occurs. In addition, at an observation time of approximately 40 hours a small bump in the graph is visible. Most probably, at that time a considerable amount of water is reaching the sensor dielectrics, which leads to a pronounced rise of the measured capacitance. With advancing time, a further non-linear gain in signal has been recorded, reflecting the behavior of the composite humidity sensor when directly exposed to water.

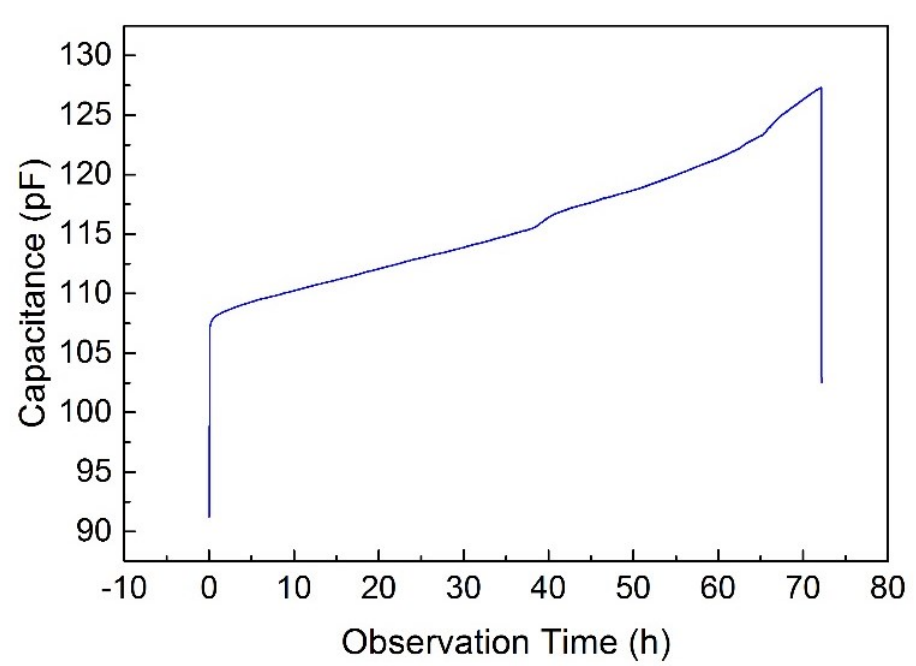

Figure 9: Capacitance of a GF-PA6 sample with integrated humidity sensor during water immersion for a period of more than 72 hours 
To get some more inside in the water diffusion properties of the glass fiber-reinforced thermoplastic GFPA6, the water uptake of a respective sample without humidity sensor has been determined. Commonly, Fick's second law (equation (1)) is used to describe diffusion in a system at which an accumulation of the penetrant (e.g. water) in the material takes place [12]. Equation (1) is giving the change of concentration with time at a certain position in the material. $D$ is the diffusion coefficient.

$$
\frac{\partial c}{\partial t}=\frac{\partial}{\partial x}\left[D \frac{\partial c}{\partial x}\right]
$$

Based on Fick's second law, equation (2) was derived to study the sorption kinetics, more precise, the diffusion of small molecules in polymers [13, 14].

$$
\frac{c_{t}}{c_{\text {inf }}}=1-\frac{4}{\pi} \sum_{n=0}^{\infty} \frac{(-1)^{n}}{2 n+1} \exp \left[\frac{-D(2 n+1)^{2} \pi^{2} t}{4 l^{2}}\right] \times \cos \left[\frac{(2 n+1) \pi x}{2 l}\right]
$$

Assuming a sample of thickness $L=21$ is being immersed in an infinite bath of penetrant (e.g. water), equation (2) is giving the concentration of the penetrant $c_{t}$ at time $t$ at any vertical position $x$ within the material relative to the saturation concentration Cinf. Finally, integrating equation (2) is giving the mass of the absorbed penetrant $m_{t}$ related to the highest possible (saturation) mass $m_{\text {inf }}$ that can be approximated for short observation times with equation (3) [12].

$$
\frac{m_{t}}{m_{\text {inf }}}=\frac{2}{l}\left(\frac{D}{\pi}\right)^{0.5} t^{0.5}
$$

This ratio can be related to Figure 10 in which the gain in mass due to water absorption for GF-PA6 is depicted. As the mass increase is displayed as specified by equation (3), the diffusion coefficient $D$ can be extracted directly from the graph. The thickness of the laminate $(L=2 I)$ is $640 \mu \mathrm{m}$. According to a mean slope of $1.3 \cdot 10^{-3} \mathrm{~s}^{-0.5}$, we can determine a diffusion coefficient $D$ of $1.3 \cdot 10^{-13} \mathrm{~m}^{2} / \mathrm{s}$ for the GFPA6 with 39 vol. \% of glass fibers.

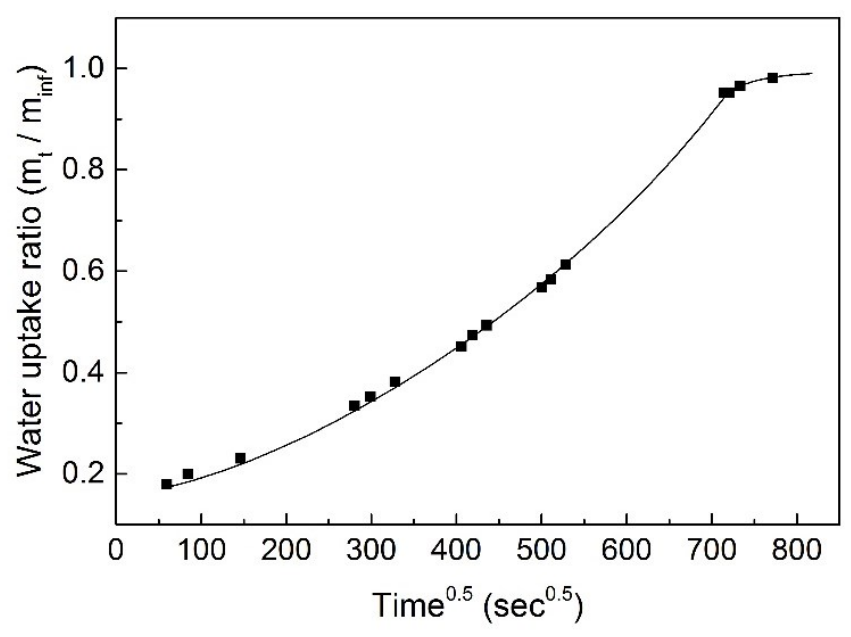

Figure 10: Water up-take of GF-PA6 as function of immersion time

With this value for the diffusion constant $D$, concentration profiles for different immersion times have been calculated according to equation (2). The resulting graphs are shown in Figure 11 together with a cross-section of the laminate. In the image of the cross-section, the edge of the Kapton $₫$ substrate is clearly visible, indicating a sensor position at $170 \mu \mathrm{m}$ from the left surface. 

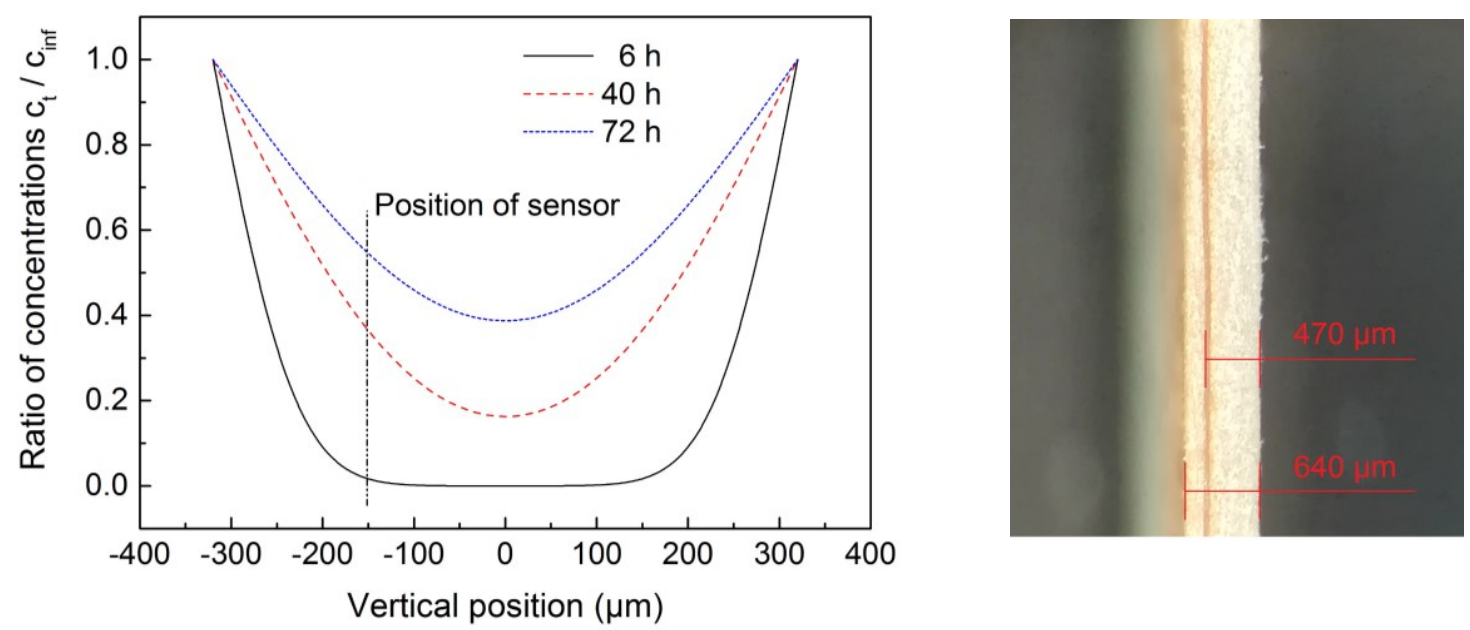

Figure 11: Calculated concentration profiles of water in GF-PA6 for different diffusion times (left) and cross section of laminate with embedded sensor substrate (Kapton $\circledast$ CR foil)

After a time of 6 hours, almost no water is reaching the humidity sensor. Since the sensor was displaying an increase in capacitance from the beginning of water immersion (see Figure 8), obviously the embedded sensor is capable of detecting water also outside the sensitive dielectrics, which is in agreement with the results of the FE simulation. With advancing time, more and more water is penetrating into the composite dielectrics too, leading to strong growth in capacitance. Thus by combining diffusion profiles of Figure 11 with capacitance values of Figures 8 or 9, a local determination of the actual water content in the laminate should be possible.

\section{Conclusion}

By integrating capacitive humidity sensors into fiber-reinforced plastic GF-PA6 during fabrication, we have demonstrated the monitoring of water uptake by the material during water storage. The very sensitive micro-composite dielectric as well as the current sensor layout ensures a detection of smallest amounts of water inside the laminate. From the sensor characteristics, it is obvious that already a mean water uptake of approx. $0.5 \mathrm{wt}$. \% can be determined reliably. After calibration of the sensor according to the responsivity to water and diffusion profiles of respective fiber-reinforced plastics, a monitoring of lateral and vertical humidity distributions is possible. Although water monitoring was demonstrated with a thermoplastic glass fiber-reinforced laminate, the technology is not limited to this type of material. The substrate foil and the binder of the nano- or microcomposite layer can be adapted to different host materials, thus a sensor integration would be also possible in thermosetting resins, for instance. However, appropriate strength tests have to be carried out for each material in order to ensure the mechanical integrity of the component.

\section{Acknowledgements}

This work was performed within the Federal Cluster of Excellence EXC 1075 "MERGE Technologies for Multifunctional Lightweight Structures", which is funded by the German Research Foundation (DFG). Financial support is gratefully acknowledged.

\section{References}

[1] Selzer, R.; Friedrich, K.: Influence of water up-take on interlaminar fracture properties of carbon fibre-reinforced polymer composites. J. Mat. Science, 30 (1995), pp. 334-338.

doi: 10.1007/BF00354392 
[2] Gellert, E.P.; Turley, D.M.: Seawater immersion ageing of glass-fibre reinforced polymer laminates for marine applications. Composites: Part A, 30 (1999), pp. 1259-1265. doi: 10.1016/S1359-835X(99)00037-8

[3] Rittersma, Z.M.: Recent achievements in miniaturised humidity sensors - review of transduction techniques. Sensors and Actuators A, 96 (2002), pp. 196-210. doi: 10.1016/S0924-4247(01)00788-9

[4] Yamazoe, N.; Shimizu, Y.: Humidity Sensors: Principles and Applications. Sens. and Actuators, 10 (1986), p. 379. doi: 10.1016/0250-6874(86)80055-5

[5] Mraovic, M.; Muck, T.; Pivar, M.; Trontelj, J.; Pletersek, A.: Humidity Sensors Printed on Recycled Paper and Cardboard. Sens., 14 (2014), pp. 13628-13643. doi: 10.3390/s140813628

[6] Hammacher, J.; Saettler, P.; Kreyßig, K.; Martin, J.; Seiler, B.; Michel, B.: Influence of nano composites on reliability and mechanical properties of moisture sensors. In: Proceedings of Nanofair Conference 2009 (2009).

[7] Piasta, D.; Martin, J.; Wegener, M.; Hammacher, J.; Geßner, T.: High sensitive humidity sensors based on nanocomposites. In: Proceedings of Smart Systems Integration Conference 2010, SSI 2010 (2010).

[8] Seider, T.; Martin, J.; Boeddicker, A.; Rühling, J.; Wett, D.; Nestler, D.; Wagner, G.; Huebler, A.C.; Otto, T.; Gessner, T.: Highly-sensitive humidity sensors for condition monitoring of hybrid laminates. Mat. Sci. Forum, 825-826 (2015), pp. 579-585. doi: 10.4028/www.scientific.net/MSF.825-826.579

[9] Si, J.J.; Ono, H.; Uchida, K.; Nozaki, S.; Morisaki, H.; Itoh, N. : Correlation between the dielectric constant and porosity of nanoporous silica thin films deposited by gas evaporation technique. Appl. Phys. Lett., 79 (2001), pp. 3140-3142. doi: 10.1063/1.1415042

[10] Tuncer, E.; Sauers, I.; James, D.R.; Ellis, A.R.: Dielectric properties of polyvinyl alcohol, poly(methyl methacrylate), polyvinyl butyral resin and polyimide at low temperatures. In: AIP Conference Proceedings, 986 (2008), pp. 190-195. doi: 10.1063/1.2900345

[11] Benchirouf, A.; Palaniyappan, S.; Ramalingame, R.; Raghunandan, P.; Jagemann, T.; Müller, C.; Hietschold, M.; Kanoun, O.: Electrical Properties of Multi-Walled Carbon Nanotubes/PEDOT:PSS Nanocomposites Thin Films under Temperature and Humidity Effects. Sens. and Actuators B: Chem., 224 (2016), 344-350. doi: 10.1016/j.snb.2015.10.009

[12] Karimi, M.: Diffusion in Polymer Solids and Solutions. In: Mass Transfer in Chemical Engineering Processes, Ed. Jozef Markos, InTech (2011). doi: 10.5772/23436

[13] Crank, J.: The Mathematics of Diffusion (Second Edition). Oxford: Claredon Press, 1975.

[14] Comyn, J.: Introduction to Polymer Permeability and the Mathematics of Diffusion, In: Comyn, J. (ed.). Polymer Permeability, London: Elsevier Applied Science Northern Ireland, 1985, pp. 1-10. 